\title{
Asymptotic analysis of turbulent boundary-layer flow of purely viscous non-Newtonian fluids
}

\author{
J.B.R. Loureiro ${ }^{\mathrm{a}, \mathrm{b}, *}$, A.P. Silva Freire ${ }^{\mathrm{a}}$ \\ ${ }^{a}$ Mechanical Engineering Program (PEM/COPPE/UFRJ), C.P. 68503, 21941-972 Rio de Janeiro, Brazil \\ ${ }^{\mathrm{b}}$ Mechanical Engineering Department (DEM/Poli/UFRJ), C.P. 68503, 21941-972 Rio de Janeiro, Brazil
}

\section{A R T I C L E I N F O}

\section{Article history:}

Received 17 August 2012

Received in revised form 13 May 2013

Accepted 20 May 2013

Available online 1 June 2013

\section{Keywords:}

Asymptotic structure

Turbulent boundary layer

Non-Newtonian

\begin{abstract}
A B S T R A C T
The asymptotic structure of turbulent boundary layers of purely viscous non-Newtonian systems is investigated through the intermediate variable technique. The cases of power-law and Carreau fluids are discussed in detail. Results show that a classical two-layered structure persists, with a viscous layer thickness that is dependent on the power-law index, $n$, and a logarithmic solution in the fully turbulent region. For Carreau fluids, in general, a three-layered structure emerges, with two nested viscous sub-layers. Experimental and numerical data from other authors are used to determine the functional behaviour of the linear coefficient of the log-law with $n$.
\end{abstract}

(c) 2013 Elsevier B.V. All rights reserved.

\section{Introduction}

The intricacies of the structure of the attached turbulent boundary layer of a Newtonian fluid were a subject of renewed interest in the sixties. Important aspects of the problem were given fresh vistas inspired by new visualization and signal processing techniques. Possibly stimulated by the results of Townsend [1] and Grant [2], which suggested the existence of long structures in the streamwise direction, many authors, notably Kline et al. [3] and Kovasznay [4], proposed physical models to explain the interactions between the different flow scales. The notion that the turbulent boundary layer consists, on one hand, of a slow convective motion of large eddies with sizes comparable to its thickness and much larger than the energy containing eddies and, on the other hand, of low-speed streaks in the near wall region, produced a picture that was identified with a double structure.

In fact, Kovasznay [4] proposed the turbulent boundary layer to be subdivided into four distinct flow regions: a viscous sublayer, a wall-turbulent layer, an outer turbulent region and an outer edge interface (superlayer). Of course, the two-layer concept coincided with some early dimensional arguments by Prandtl [5] and von Kármán [6] and the establishment of a flow structure dominated by regions where (i) the turbulent and laminar stresses have the

\footnotetext{
* Corresponding author at: Mechanical Engineering Program (PEM/COPPE/UFRJ) C.P. 68503 , 21941-972, Rio de Janeiro, Brazil. Tel.: +55 2136223548; fax: +55 2136223552.

E-mail addresses: jbrloureiro@gmail.com, jbrloureiro@mecanica.coppe.ufrj.br (J.B.R. Loureiro).
}

same magnitude and (ii) the outer velocity profile can be expressed as a small perturbation to the external flow solution.

From the view point of perturbation methods, the above concepts were reconciled for the first time by Yajnik [7] through the matched asymptotic expansions method. His analysis did not resort to any closure hypothesis, but to hypotheses describing the order of magnitude of the various terms in the equations of motion in the limit as Reynolds number approaches infinity. The general asymptotic structure deduced by Yajnik [7] was soon confirmed through contributions of Mellor [8] and Bush and Fendell [9].

In a different approach to the problem, Sychev and Sychev [10], Cruz and Silva Freire [11] and Loureiro and Silva Freire [12] gave a new interpretation to the flow relevant stretching parameters, highlighting the importance of a region where inertia forces, pressure and turbulent friction forces have the same order of magnitude. The works of Cruz and Silva Freire [11] and Loureiro and Silva Freire [12] used the intermediate variable technique introduced by Kaplun [13] and Lagerstrom and Casten [14]. Intermediate variables and the notion of limit process are the fundamental tools in perturbation techniques.

The purpose of the present work is to discuss the asymptotic structure of the turbulent boundary layer of purely viscous nonNewtonian fluids. In this context, we discuss the flow structure for fluids whose relation between viscosity and flow shear rate can be expressed in terms of simple equations. The special cases of power-law and Carreau fluids are detailed. The general conclusion is that for a power-law fluid the two-layered structure of the turbulent boundary layer is not altered by changes in the viscosity model. Modifications result just from local changes in the thickness and solution of the viscous region. In particular, a 


\begin{tabular}{|c|c|c|c|}
\hline \multicolumn{4}{|c|}{ Nomenclature } \\
\hline$a$ & parameter in the Carreau-Yasuda viscosity model & $\hat{\epsilon}$ & small parameter $\left(=\left(1 /\left(\epsilon^{2} R_{e}\right)\right)^{1 / n}\right)$ \\
\hline$A_{n}, B_{n}$ & parameters in the law of the wall & $\tilde{\epsilon}$ & small parameter $\left(=\hat{\epsilon}^{n}\right)$ \\
\hline & pipe diameter & $\eta$ & function in $\Xi$ \\
\hline$R$ & pipe radius & $x$ & von Karman's constant $(=0.4)$ \\
\hline E & ordinary differential equation of variables $x, y$ and $\epsilon$ & $\lambda$ & parameter in Carreau-Yasuda model \\
\hline$f$ & friction coefficient & $\mu$ & viscosity \\
\hline$f, F$ & function of variables $x, y$ and small parameter $\epsilon$ & $v$ & kinematic viscosity \\
\hline \multirow[t]{2}{*}{$f_{2}$} & function describing the similarity dependence in the & $\theta$ & parameter in Carreau model \\
\hline & laminar sublayer & $\rho$ & fluid density \\
\hline$g, G$ & function of variables $x, y$ and small parameter $\epsilon$ & $\tau$ & shear stress \\
\hline$g_{1}$ & function describing the external flow behaviour & $\xi$ & $=y / R$ \\
\hline K & consistency index in power-law model & $\Xi$ & space of all positive continuous functions on $(0,1]$ \\
\hline l & mixing-length & & \\
\hline$\ell$ & wall region characteristic length & \multicolumn{2}{|c|}{ Subscripts } \\
\hline $\begin{array}{l}n \\
n^{\prime}\end{array}$ & $\begin{array}{l}\text { index in power-law or Carreau models } \\
\text { index in Carreau-Yasuda models }\end{array}$ & 0 & $\begin{array}{l}\text { zero-shear viscosity in Carreau or Carreau-Yasuda mod- } \\
\text { els }\end{array}$ \\
\hline$N_{D}$ & Reynolds number $\left(=\rho U^{2-n} D^{n} / K\right)$ & 1,2 & transformed equation upon passage of $\eta$-limit \\
\hline$p$ & pressure & $\eta$ & intermediate variable or function, passage of $\eta$-limit \\
\hline$r$ & radial distance & $p$ & power-law condition \\
\hline$R_{e}$ & Reynolds number $\left(=\rho U_{\infty}^{2-n} L^{n} / K\right)$ & $u$ & longitudinal velocity \\
\hline$U, u$ & longitudinal velocity component in a Cartesian system & $w$ & wall condition \\
\hline$u_{*}$ & friction velocity & $\infty$ & external flow condition \\
\hline$V, v$ & transversal velocity component in a Cartesian system & $\infty$ & infinity shear viscosity in Carreau or Carreau-Yasuda \\
\hline$x, y$ & flow Cartesian coordinates & & models \\
\hline & wall region similarity variable $(=y / \ell)$ & & \\
\hline \multirow[t]{2}{*}{$Z$} & \multirow[t]{2}{*}{ Reynolds number $\left(=R^{n} \rho\left(u_{*}\right)^{2-n} / K\right)$} & \multicolumn{2}{|c|}{ Superscripts } \\
\hline & & & fluctuating quantity \\
\hline \multicolumn{2}{|c|}{ Greek symbols } & $\sim$ & intermediate variable or function \\
\hline$\alpha$ & parameter in Carreau model $\left(=\left(\left(v_{0} / v_{\infty}\right)-1\right)\right)$ & $\wedge$ & intermediate variable or function \\
\hline$\underset{\tilde{s}}{\beta \beta}$ & parameter in Carreau model $\left(=\left(\left(U_{\infty} / L\right) \theta\right)^{n-1}\right)$ & - & mean quantity \\
\hline$\delta$ & turbulent layer thickness & & \\
\hline$\hat{\delta}$ & viscous layer thickness & & \\
\hline$\epsilon$ & small parameter $\left(=u_{*} / U_{\infty}\right)$ & & \\
\hline
\end{tabular}

logarithmic solution is always observed in the fully turbulent region. For Carreau fluids, in general, a three-layered structure emerges, with two nested viscous sub-layers. This result is reported here for the first time. For some particular limiting cases, however, different structures may result.

A comparison between the present results and the data of other authors is made to determine the functional dependence of the log-law linear coefficient on the power-law index, $n$. The angular coefficient is suggested to be independent of $n$ and equal to 2.5 $\left(=\chi^{-1}\right.$, the inverse of von Kármán's constant), whereas the linear coefficient is suggested to vary according to a power-law expression, the reciprocal of $n$.

Some authors have used pressure drop measurements to investigate the form of the velocity profile. Bogue and Metzner [15], however, have pointed out that the use of friction data is an insensitive way to find information on the velocity profiles, since only an integrated effect is revealed. For this reason, a direct analysis is preferred in the present work. Data furnished by the experiments of Bogue and Metzner [15], Escudier and Presti [16], Pereira and Pinho [17] and Japper-Jaafar et al. [18] and the DNS data of Rudman et al. [19] are used to develop local analysis of the mean velocity profiles.

\section{Early work}

The analysis of turbulent flow of non-Newtonian fluids over smooth walls received a classical treatment in the fifties by Metzner and Reed [20] and Dodge and Metzner [21].
In the former work, global conservation principles were used to develop expressions for the rate of shear of a fluid that are independent of the fluid properties provided the fluids were purely viscous and time-independent. The analysis naturally led to the introduction of a generalized Reynolds number that was used to correlate the available data in a friction-factor/Reynolds-number chart. In the logarithmic chart, the data covered ten decades, ranging from $10^{-4}$ to $10^{5}$.

The generalized Reynolds number is defined as

$N_{R}=\frac{D^{n} U^{2-n} \rho}{K}$,

where the symbols follow their conventional meaning, $D=$ pipe diameter, $U=$ mean flow velocity, $\rho=$ fluid density, and $K$ and $n$ are defined through Eq. (2),

$\tau=K\left|\frac{d u}{d y}\right|^{n-1}\left(\frac{d u}{d y}\right)=\mu\left(\frac{d u}{d y}\right)$.

The latter work was entirely devoted to the study of turbulent flows. Dimensional arguments similar to those first employed by Millikan [22] were used to find a local solution for the mean velocity profile that was supposed to be valid in the turbulent core of pipe flows.

In the laminar sublayer, the local solution of a power-law fluid was written as

$\frac{u}{u_{*}}=f_{2}\left(Z \xi^{n}, n\right)$, 
where

$Z=R^{n} \rho\left(u_{*}\right)^{2-n} / K, \quad \xi=y / R$,

and $u_{*}$ is the friction velocity $\left(=\sqrt{\tau_{w} / \rho}\right)$.

Considerations regarding the overlap of the turbulent solution with the purely viscous solution led to

$\frac{u}{u_{*}}=A_{n} \ln Z \xi^{n}+B_{n}-g_{1}(\xi, n)$,

where $g_{1}$ is a function that must accommodate the external flow behaviour and $A_{n}$ and $B_{n}$ must be determined experimentally.

Measurements of frictional pressure-loss were then used to determine the coefficients in Eq. (5), yielding

$$
\begin{aligned}
\frac{u}{u_{*}}= & \frac{5.66}{n^{0.75}} \log y^{+}-\frac{0.40}{n^{1.2}} \\
& +\frac{2.458}{n^{0.75}}\left[1.960+1.255 n-1.628 n \log \left(3+\frac{1}{n}\right)\right],
\end{aligned}
$$

with $u^{+}=u / u_{*}, y^{+}=Z \xi^{n}$.

Some coefficients in Eq. (6) were subsequently corrected in an errata and in [23]. Skelland [23] only rectified mistakes that had resulted from faulty algebraic manipulations. The analysis essentially followed the theoretical treatment of Dodge and Metzner [21], considering the slope of the log-term to vary with $n^{-0.75}$. Subsequent work by Bogue and Metzner [15] and Clapp [24] showed this dependence to be on $n^{-1}$ (in relation to their definition of $y^{+}$). Therefore, the expressions presented in Dodge and Metzner [21] and Skelland [23] should not be used.

Appropriate equations for the fully turbulent velocity profile and the friction coefficient are introduced here in the following developments.

\section{Viscous layer solution}

The viscous layer solution for a power-law fluid can be found from a simple integration of

$\frac{\partial \tau}{\partial y}=\frac{\partial}{\partial y}\left(K\left(\frac{d u}{d y}\right)^{n}\right)=0$,

resulting in the linear expression,

$\frac{u}{u_{*}}=\frac{y}{\left(K u_{*}^{n-2} / \rho\right)^{1 / n}}$,

or else, using the usual Newtonian notation,

$u^{+}=y^{+}$.

Thus, it is apparent from Eq. (8) that the wall layer relevant length scale is

$\ell=\left(K u_{*}^{n-2} / \rho\right)^{1 / n}$,

and that the apparent viscosity used in the definition of $y^{+}$is the wall viscosity.

Dodge and Metzner [21] instead defined in their work $u^{+}=\left(y^{+}\right)^{1 / n}$, implying that $y^{+}=Z \xi^{n}=y^{n}\left(u_{*}\right)^{2-n} \rho / K$. In the present work, we stick to the definition of Eq. (8).

\section{Turbulent layer solution: dimensional arguments}

If there is to be a logarithmic solution in the inner regions of a flow and, if this region is to comply to similarity conditions, then it is clear that the similarity length and velocity scales must be $\ell$ and $u_{*}$ as implied by Eq. ( 8 ).

Therefore, a log-solution should be written as
$\frac{u}{u_{*}}=A_{n} \ln \left(\frac{y}{\ell}\right)+B_{n}-g_{1}(\xi, n)$,

and not as Eq. (5).

The implication is simple for if Eq. (5) is to be written in terms of the inner variables of Dodge and Metzner [21], Eq. (4), it should read as

$\frac{u}{u_{*}}=\frac{A_{n}}{n} \ln \left(\frac{y}{\ell}\right)^{n}+B_{n}-g_{1}(\xi, n)=\frac{A_{n}}{n} \ln Z \xi^{n}+B_{n}-g_{1}(\xi, n)$.

There is, thus, cause to believe that the slope of the log-term in Eq. (6) should vary with the reciprocal of $n$ and not with the inverse of $n^{0.75}$.

This fact was very early noticed by Clapp [24] and Bogue and Metzner [15], who proposed mean velocity profile formulations with this form, the reciprocal of $n$. The more limited extent of their experimental work, as compared to Dodge and Metzner's, may be a possible explanation for the recurrent use of Eq. (6) in literature.

\section{Turbulent layer solution: analytical suggestion for the existence of the log-law}

Consider as a starting point the $x$-boundary layer equation for a power-law fluid, that is,

$\rho u \frac{\partial u}{\partial x}+\rho v \frac{\partial u}{\partial y}=-\frac{\partial p}{\partial x}+\frac{\partial}{\partial y}\left(K\left(\frac{\partial u}{\partial y}\right)^{n}\right)$.

Consider next that all variables can be decomposed into mean and fluctuating parts, e.g.,

$u=\bar{u}+u^{\prime}$

and that the binomial expansion can be used with $\left|u^{\prime} / \bar{u}\right| \ll 1$, that is,

$\left(\bar{u}+u^{\prime}\right)^{n}=\bar{u}^{n}+n \bar{u}^{(n-1)} u^{\prime}+\frac{n(n-1)}{2} \bar{u}^{(n-2)} u^{\prime 2}+e s t$.

Substitution of Eqs. (14) and (15) in Eq. (13), results

$$
\begin{aligned}
\rho \bar{u} \frac{\partial \bar{u}}{\partial x}+\rho \bar{v} \frac{\partial \bar{u}}{\partial y}= & -\frac{\partial \bar{p}}{\partial x}-\rho \frac{\partial \overline{u^{\prime} v^{\prime}}}{\partial y} \\
& +\frac{\partial}{\partial y}\left(K\left(\frac{\partial \bar{u}}{\partial y}\right)^{n}+K \frac{n(n-1)}{2}\left(\frac{\partial \bar{u}}{\partial y}\right)^{n-2} \overline{\left(\frac{\partial u^{\prime}}{\partial y}\right)^{2}}\right) .
\end{aligned}
$$

The usual procedure to find a local solution in the fully turbulent region would be to consider the boundary layer to have a classical two-layered structure. Then, selection of the turbulent terms in Eq. (16) naturally leads to

$-\rho \frac{\partial \overline{u^{\prime} v^{\prime}}}{\partial y}+\frac{\partial}{\partial y}\left(K \frac{n(n-1)}{2}\left(\frac{\partial \bar{u}}{\partial y}\right)^{n-2} \overline{\left(\frac{\partial u^{\prime}}{\partial y}\right)^{2}}\right)=0$.

In fact, we show in the next section through a proper asymptotic analysis that Eq. (17) makes no sense since, formally, the gradient of the mean viscous stress cannot be neglected. At this stage, however, the lack of information about the suitable stretching variables and the relative order of magnitude between the mean and fluctuating quantities prevents us from performing any consistent analysis.

To further proceed it is convenient to close the turbulent terms. The simplest possible manner is to consider the order of magnitude of the fluctuations to be the same in the fully turbulent region and proportional to the local velocity gradient (mixing-length hypothesis), so that,

$\operatorname{order}\left(u^{\prime}\right)=\operatorname{order}\left(v^{\prime}\right)=l \frac{\partial \bar{u}}{\partial y}$. 
The above consideration concerning the mixing-length hypothesis is not of crucial importance to the following developments, it has been used here for simplicity. This type of approach is not new; e.g., early works by Clapp [24] and Tomita [25] applied the mixing-length concept to non-Newtonian fluids to find a relation between the friction coefficient and the generalized Reynolds number.

Substitution of Eq. (18) onto Eq. (17) yields:

$\rho \frac{\partial}{\partial y}\left(l^{2}\left(\frac{\partial \bar{u}}{\partial y}\right)^{2}\right)+K \frac{n(n-1)}{2} \frac{\partial}{\partial y}\left(\left(\frac{\partial \bar{u}}{\partial y}\right)^{n-2}\left(\frac{\partial}{\partial y}\left(l \frac{\partial \bar{u}}{\partial y}\right)\right)^{2}\right)$

$$
=0 \text {. }
$$

If the mixing-length, $l$, is then considered to be proportional to the wall distance, $l=x y$, with $x$ a constant to be determined, it follows immediately that Eq. (19) accepts a logarithmic solution.

\section{Perturbation analysis: morphological structure}

To find the asymptotic structure of the turbulent boundary layer of a non-Newtonian power-law fluid, consider Eq. (16). Consider further that all lengths are non-dimensionalized by a typical body dimension, $L$, velocities by $U_{\infty}$ and kinematic pressure by $U_{\infty}^{2}$, so that Eq. (16) can be cast in a non-dimensional form through

$$
\begin{aligned}
\bar{u} \frac{\partial \bar{u}}{\partial x}+\bar{v} \frac{\partial \bar{u}}{\partial y}= & -\frac{\partial \bar{p}}{\partial x}-\epsilon^{2} \frac{\partial \overline{u^{\prime} v^{\prime}}}{\partial y}+\epsilon^{2} \hat{\epsilon}^{n} \\
& \times \frac{\partial}{\partial y}\left(\left(\frac{\partial \bar{u}}{\partial y}\right)^{n}+\frac{n(n-1)}{2}\left(\frac{\partial \bar{u}}{\partial y}\right)^{n-2} \overline{\left(\frac{\partial u^{\prime}}{\partial y}\right)^{2}}\right)
\end{aligned}
$$

where

$\epsilon=\frac{u_{*}}{U_{\infty}}, \quad \epsilon^{2} \hat{\epsilon}^{n}=\frac{1}{R_{e}}, \quad R_{e}=\frac{\rho U_{\infty}^{2-n} L^{n}}{K}$,

and the leading order velocity fluctuations are considered to be of the order of the friction velocity.

The asymptotic structure of the flow will be determined through the single limit concept of Kaplun. The fundamental notions on perturbation methods to be used henceforth were laid down by Kaplun [13], Lagerstrom and Casten [14] and Lagerstrom [26] in extensive texts. More recent contributions can be found in Cruz and Silva Freire [11] and Loureiro and Silva Freire [12]. In particular, the article of Loureiro and Silva Freire [12] discusses in detail most of the relevant definitions and results.

To keep the present work within a permissible level of understanding, only the essential information will be repeated here. The topology on the collection of order classes introduced by Meyer [27] is used. For positive, continuous functions of a single variable $\epsilon$ defined on $(0,1]$, ord $\eta$ denotes the class of equivalence introduced in Meyer.

Definition 1. (Lagerstrom [26]). We say that $f(x, \epsilon)$ is an approximation to $g(x, \epsilon)$ uniformly valid to order $\delta(\epsilon)$ in a convex set $D(f$ is a $\delta$-approximation to $g$ ), if

$\lim (f(x, \epsilon)-g(x, \epsilon)) / \delta(\epsilon)=0, \quad \epsilon \rightarrow 0$, uniformly for $x$ in $D$.

Consider

$x_{\eta}=x / \eta(\epsilon), \quad G\left(x_{\eta} ; \epsilon\right)=F(x ; \epsilon)$,

with $\eta(\epsilon)$ defined in $\Xi$ (=space of all positive continuous functions on $(0,1])$.

Definition 2. (of Kaplun limit)(Meyer [27]). If the function $G\left(x_{\eta} ;+0\right)=\lim G\left(x_{\eta} ; \epsilon\right), \epsilon \rightarrow 0$, exists uniformly on $\left\{x_{\eta} /\left|x_{\eta}\right|>0\right\}$; then we define $\lim _{\eta} F(x ; \epsilon)=G\left(x_{\eta} ;+0\right)$.
The definition of $\eta$-limit of a function and of domains of validity were given an analogous concept for equations by Lagerstrom and Casten [14]. They made the following definitions.

Definition 3. (Lagerstrom and Casten [14]). If $E$ is an equation and $\lim _{\eta_{1}} E=E_{1}, \lim _{\eta_{2}} E=E_{2}$ and also $\lim _{\eta_{2}} E_{1}=E_{2}$, we say that $E_{1}$ contains $E_{2}$ (relative to $E$ ).

Definition 4. (Lagerstrom and Casten [14]). The formal domain of validity of an equation $F$, relative to the "full" equation $E$, is the ord $\eta$ such that $\lim _{\eta} E$ is either $F$ or an equation contained in $F$.

The above definitions naturally imply the existence of distinguished equations, obtained from specific choices of $\eta$. These equations are, in the sense of Kaplun [13], "rich" equations. A more elaborate statement is given by

Definition 5. An equation $P$ that contains other limit equations but is not contained by any other is said to be a principal equation.

An equation which is not principal is said to be an intermediate equation.

The previous definitions are complemented by the following statement:

Principle (Kaplun [13]). If $y$ is a solution of an equation $E$ and $E^{*}$ is an approximate equation, then there exists a solution $y^{*}$ of $E^{*}$ whose actual domain of validity (as an approximation to $y$ ) includes the formal domain of validity of $E^{*}$ (as an approximation to $E$ ).

To analyze the turbulent boundary layer, make

$y_{\eta}=y / \eta(\epsilon, \hat{\epsilon}), \quad \widehat{u}_{i}\left(y_{\eta} ; \epsilon, \hat{\epsilon}\right)=u_{i}(y ; \epsilon, \hat{\epsilon})$,

with $\eta(\epsilon, \hat{\epsilon})$ a function defined in $\Xi$ (=space of all positive continuous functions on $(0,1])$.

Upon substitution of Eq. (24) into Eq. (20) and considering that close to the wall $O(\bar{u})=O\left(u^{\prime}\right)$, the following formal limits are found depending on the order class of $\eta$ :

$\operatorname{ord} \eta=\operatorname{ord} 1: \quad \overline{\hat{u}} \frac{\partial \overline{\hat{u}}}{\partial x}+\overline{\hat{v}} \frac{\partial \overline{\hat{u}}}{\partial y_{\eta}}=-\frac{\partial \overline{\hat{p}}}{\partial x}$

ord $\epsilon^{2}<$ ord $\eta<$ ord 1: $\quad \overline{\hat{u}} \frac{\partial \overline{\hat{u}}}{\partial x}+\overline{\hat{v}} \frac{\partial \overline{\hat{u}}}{\partial y_{\eta}}=-\frac{\partial \overline{\hat{p}}}{\partial x}$,

$\operatorname{ord} \epsilon^{2}=\operatorname{ord} \eta: \quad \overline{\hat{u}} \frac{\partial \overline{\hat{u}}}{\partial x}+\overline{\hat{v}} \frac{\partial \overline{\hat{u}}}{\partial y_{\eta}}=-\frac{\partial \overline{\hat{p}}}{\partial x}-\frac{\partial \overline{\hat{u}}^{\prime} \hat{v}^{\prime}}{\partial y_{\eta}}$

ord $(\epsilon \hat{\epsilon})<$ ord $\eta<$ ord $\epsilon^{2}: \quad \frac{\partial \overline{\hat{u}^{\prime} \hat{v}^{\prime}}}{\partial y_{\eta}}=0$

$\operatorname{ord}(\epsilon \hat{\epsilon})=\operatorname{ord} \eta: \quad-\frac{\partial \bar{u}^{\prime} \hat{v}^{\prime}}{\partial y_{\eta}}+\frac{\partial}{\partial y_{\eta}}\left(\left(\frac{\partial \hat{\hat{u}}}{\partial y_{\eta}}\right)^{n}+\frac{n(n-1)}{2}\left(\frac{\partial \overline{\hat{u}}}{\partial y_{\eta}}\right)^{n-2} \overline{\left(\frac{\partial \hat{u}^{\prime}}{\partial y_{\eta}}\right)^{2}}\right)=0$

ord $\eta<\operatorname{ord}(\epsilon \hat{\epsilon})$ :

$$
\frac{\partial}{\partial y_{\eta}}\left(\left(\frac{\partial \overline{\hat{u}}}{\partial y_{\eta}}\right)^{n}+\frac{n(n-1)}{2}\left(\frac{\partial \overline{\hat{u}}}{\partial y_{\eta}}\right)^{n-2} \overline{\left(\frac{\partial \hat{u}^{\prime}}{\partial y_{\eta}}\right)^{2}}\right)=0 .
$$

The above results show that the turbulent boundary layer of a power-law fluid exhibits a canonical two-deck structure defined by the principal equations, Eqs. (27) and (29). The viscosity of the fluid defines the thickness of the viscous region through $\epsilon \hat{\epsilon}=\left(\epsilon^{n-2} / R_{e}\right)^{1 / n}$. 
The influence of the non-Newtonian turbulence term, Eq. (29), is shown to be restrict to domain $\operatorname{ord}(\eta) \leqslant \operatorname{ord}(\epsilon \hat{\epsilon})$. The turbulence dominated region (defined by $\operatorname{ord}(\epsilon \hat{\epsilon})<\operatorname{ord}(\eta)<\operatorname{ord}\left(\epsilon^{2}\right)$ ) is governed by turbulence originated from the inertial terms in the equations of motion. No contribution arises from the averaging of the non-linear viscous terms.

In fact, in view of the above analysis, we can say that Eq. (17) makes no sense since, formally, we have seen that in the fully turbulent region, $\operatorname{ord}(\epsilon \hat{\epsilon})<\operatorname{ord}(\eta)<\operatorname{ord}\left(\epsilon^{2}\right)$,

$\operatorname{ord}\left(\frac{\partial \overline{u^{\prime} v^{\prime}}}{\partial y}\right)>\operatorname{ord}\left(\frac{\partial}{\partial y}\left(\left(\frac{\partial \bar{u}}{\partial y}\right)^{n-2} \overline{\left(\frac{\partial u^{\prime}}{\partial y}\right)^{2}}\right)\right)$

The two relevant length scales of the flow are then:

- Turbulent layer thickness, $\tilde{\delta}=\left(u_{*}^{2} / U_{\infty}^{2}\right) L$.

- Viscous sub-layer thickness, $\hat{\delta}=\left(K u_{*}^{n-2} / \rho\right)^{1 / n}$.

The obvious conclusion is that for $n<1$ the viscous sub-layer thickness increases (in relation to that of a Newtonian fluid, $n=1)$, whereas for $n>1, \hat{\delta}$ decreases.

\section{Law of the wall: power-law fluid}

The linear coefficient of Eq. (11) will be discussed next according to the experimental data of Escudier and Presti [16], Pereira and Pinho [17], Bogue and Metzner [15] and the DNS data of Rudman et al. [19].

Mean velocity profiles obtained by Escudier and Presti [16] and Pereira and Pinho [17] are shown in Fig. 1 for different types of shear-thinning fluids. Provided they are plotted in terms of $y^{+}=y \mid \ell$ they all present the same slope $\left(=\chi^{-1}\right)$ and different levels. A decrease in the value of $n$ pushes the level up as expected.

To estimate the value of $B_{n}$ in Eq. (11) the procedure adopted in Loureiro et al. [28] and Loureiro and Silva Freire [29] was repeated here. Global optimization algorithms based on direct search methods were used. Despite their tendency to converge more slowly, direct search methods are more tolerant to the presence of noise in the function and to constraints. Four different methods were used for the solution search: Nelder Mead, differential evolution, simulated annealing and random search. Only when all four methods

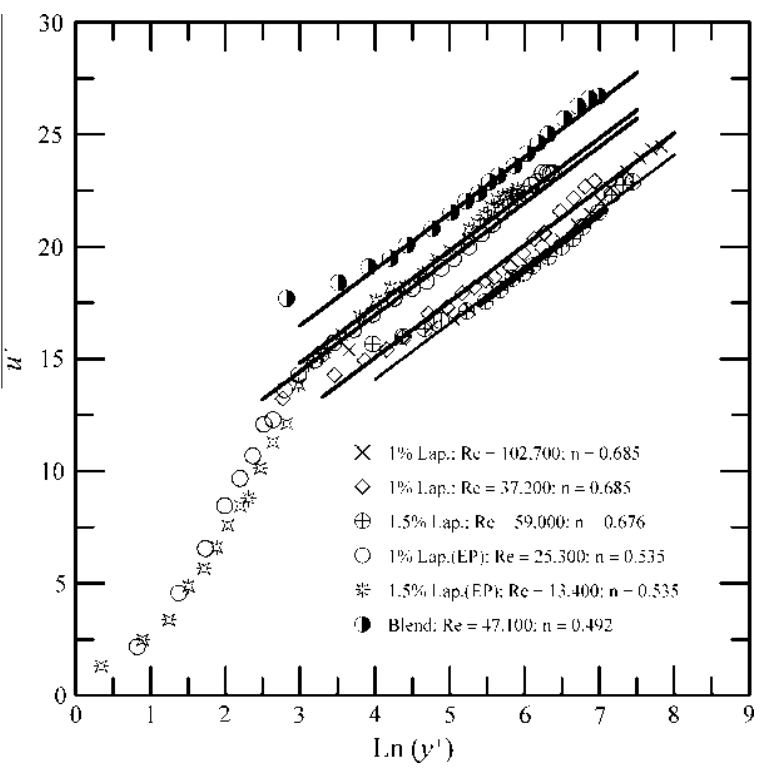

Fig. 1. Mean velocity profiles in wall coordinates according to the data of Pereira and Pinho [17] and Escudier and Presti [16]. furnished consistent results, with accuracy down to the sixth decimal place, was the search stopped.

The results are shown in Fig. 2. Clapp [24] was possibly the first to have used the velocity distribution to determine $B_{n}$. Using six velocity profiles for fluids with $n$ varying between $0.698-0.7$ (four profiles) and 0.765 (two profiles), he proposed the correlation, $B_{n}=3.8 / n$. To analyze the data in Fig. 2 we have included the point $\left(n, B_{n}\right)=(1,5)$ corresponding to Newtonian fluids and disregarded the two lowest points where $B_{n}$ is about 4 .

Three curve fittings were made: two power-law fits, $B_{n}=4.56 /$ $n^{0.755}, B_{n}=2.68 / n^{1.626}$, and a reciprocal fit, $B_{n}=4.02 / n$. In the first power-law fit, the Newtonian point was considered, resulting in a decay with the power 0.755 . Exclusion of this single point increased the decay power to 1.626 . Consideration of the reciprocal fit yielded the correlation $B_{n}=4.02 / n$, very close to that of Clapp [24].

Fig. 2 also shows the low Reynolds number DNS results of Rudman et al. [19] for $B_{n}$. A curve fit to the DNS data furnishes $B_{n}=4.92 / n$. Overall, the numerical data are shifted vertically by about $\Delta\left(B_{n}\right)=1 / n$; they do suggest, however, that $B_{n}$ varies with the reciprocal of $n$.

Bogue and Metzner [15] discussed the viscoelastic properties of alginate and Carbopol aqueous solutions, clay suspensions (Attagel), carboxymethyl cellulose (CMC) solutions and polyisobutylene in cyclohexane solutions. The conclusion was that Carbopol solutions (in low concentrations) and clays suspensions can be considered, for practical purposes, purely viscous fluids. Problems resulting from some degradation of the Carbopol between experimental runs and from the time-dependent properties of the clay suspensions until they were completely mixed were minimized through permanent rheological measurements.

The behavior of $B_{n}$ according to the data of Bogue and Metzner [15] is shown in Fig. 3. In [15], the function $B_{n}$ was presented in tabular form. The results show a strong dependence on the Reynolds number. In fact, for low Reynolds numbers ( $=5000$ and 10,000 ; shown in Fig. 3 ) changes in $B_{n}$ are as high as $54 \%$. For high Reynolds numbers $(=50,000$ and 100,000 ; not shown in Fig. 3 ), changes are much less significant, almost unnoticed at 100,000.

Two curve fits are shown in Fig. 3. For the reciprocal fit, $B_{n}=3.91 / n$, is a result that compares well with the data of Clapp [24] $\left(B_{n}=3.8 / n\right)$, Escudier and Presti [16] and Pereira and Pinho

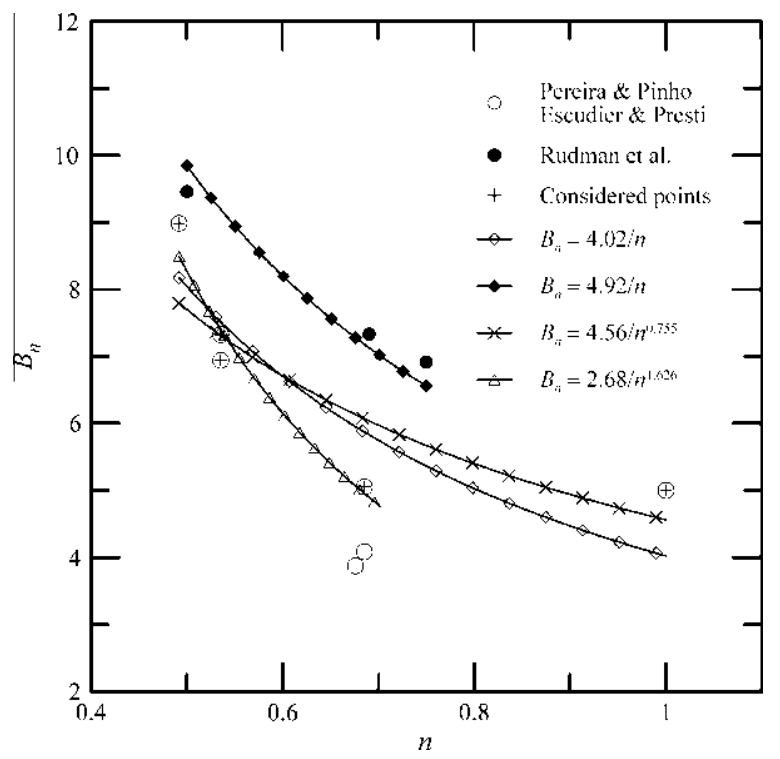

Fig. 2. Functional behaviour of the linear coefficient of the log-law with $n$ according to the data of Pereira and Pinho [17], Escudier and Presti [16] and Rudman et al. [19]. 


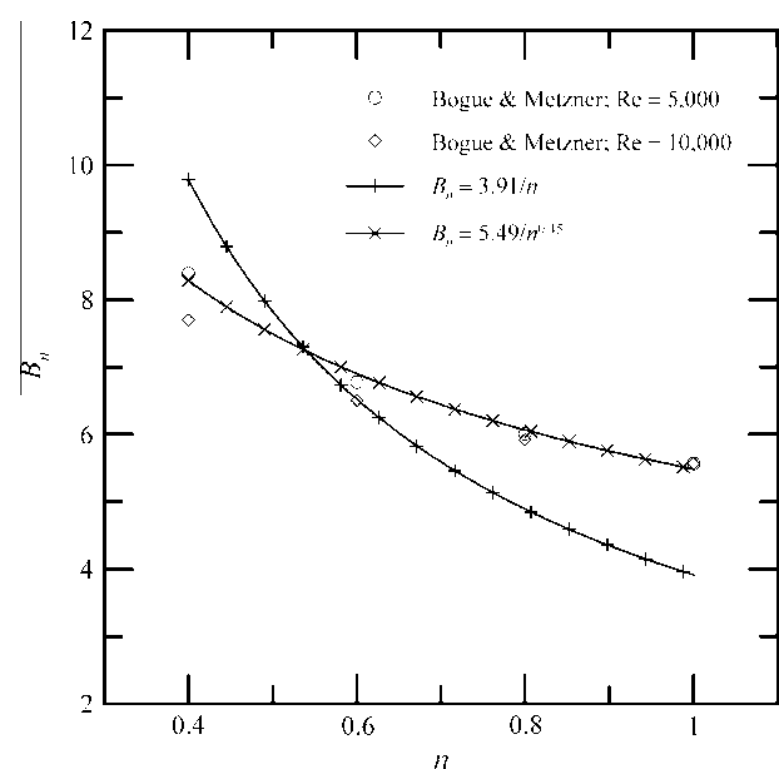

Fig. 3. Behaviour of $B_{n}$ according to the data of Bogue and Metzner [15].

[17]. For the power-law fit, $B_{n}=5.49 / n^{0.45}$, implying a result quite different from other authors.

Figs. 2 and 3 illustrate how difficult it is to disclose definite evidence from the available experimental data for purely viscous flows. The intrinsic difficulties associated with the unavoidable presence of viscoelastic effects in experiments complicates the analysis considerably.

\section{Appraisal of the above arguments for a Carreau fluid}

For a Carreau fluid the viscosity and local shear rate are related according to

$\mu=\mu_{\infty}+\left(\mu_{0}-\mu_{\infty}\right)\left(1+\left(\theta \frac{\partial u}{\partial y}\right)^{2}\right)^{\frac{n-1}{2}}$

where $\mu_{\infty}$ and $\mu_{0}$ are the limiting viscosity levels, $\theta$ is a model parameter and $n$ the power law index with $n<1$.

Considering the $x$-boundary layer equation, we can write,

$$
\begin{aligned}
\rho u \frac{\partial u}{\partial x}+\rho v \frac{\partial u}{\partial y}= & -\frac{\partial p}{\partial x} \\
& +\frac{\partial}{\partial y}\left[\left(\mu_{\infty}+\left(\mu_{0}-\mu_{\infty}\right)\left(1+\left(\theta \frac{\partial u}{\partial y}\right)^{2}\right)^{\frac{n-1}{2}}\right) \frac{\partial u}{\partial y}\right] .
\end{aligned}
$$

We again consider that all variables can be decomposed into mean and fluctuating parts, that the binomial theorem can be used and that the shear stress rate is high enough so that the unity can be neglected in Eq. (32).

The result is

$$
\begin{aligned}
\rho \bar{u} \frac{\partial \bar{u}}{\partial x}+\rho \bar{v} \frac{\partial \bar{u}}{\partial y}= & -\frac{\partial \bar{p}}{\partial x}-\rho \frac{\partial \overline{u^{\prime} v^{\prime}}}{\partial y} \\
& +\frac{\partial}{\partial y}\left(\mu_{\infty} \frac{\partial \bar{u}}{\partial y}+\left(\mu_{0}-\mu_{\infty}\right) \theta^{n-1}\left[\left(\frac{\partial \bar{u}}{\partial y}\right)^{n}+\frac{n(n-1)}{2}\left(\frac{\partial \bar{u}}{\partial y}\right)^{n-2} \overline{\left.\left.\left(\frac{\partial u^{\prime}}{\partial y}\right)^{2}\right]\right) .}\right.\right.
\end{aligned}
$$

To solve Eq. (34) in the fully turbulent region, the standard procedure requires that only the fluctuating terms be retained, that is,

$$
-\rho \frac{\partial \overline{u^{\prime} v^{\prime}}}{\partial y}+\frac{\partial}{\partial y}\left(\left(\mu_{0}-\mu_{\infty}\right) \theta^{n-1} \frac{n(n-1)}{2}\left(\frac{\partial \bar{u}}{\partial y}\right)^{n-2} \overline{\left(\frac{\partial u^{\prime}}{\partial y}\right)^{2}}\right)=0
$$

It follows immediately that considerations regarding the existence of a mixing length or any other equivalent assumption results in a logarithmic solution.

\section{Morphological structure: Carreau fluid}

For a Carreau fluid, the asymptotic structure of the turbulent boundary layer of a non-Newtonian fluid can be assessed from Eq. (36). This equation has been derived considering that all lengths are non-dimensionalized by, $L$, velocities by $U_{\infty}$ and kinematic pressure by $U_{\infty}^{2}$, so that Eq. (34) can be written as

$$
\begin{aligned}
\bar{u} \frac{\partial \bar{u}}{\partial x}+\bar{v} \frac{\partial \bar{u}}{\partial y}= & -\frac{\partial \bar{p}}{\partial x}-\epsilon^{2} \frac{\partial \overline{u^{\prime} v^{\prime}}}{\partial y}+\epsilon^{2} \tilde{\epsilon}^{n} \frac{\partial}{\partial y} \\
& \times\left(\left(\frac{\partial \bar{u}}{\partial y}\right)^{n}+\frac{n(n-1)}{2}\left(\frac{\partial \bar{u}}{\partial y}\right)^{n-2} \overline{\left(\frac{\partial u^{\prime}}{\partial y}\right)^{2}}\right)+\epsilon^{2} \hat{\epsilon}\left(\frac{\partial^{2} \bar{u}}{\partial y^{2}}\right),
\end{aligned}
$$

where

$\epsilon=\frac{u_{*}}{U_{\infty}}, \quad \epsilon^{2} \tilde{\epsilon}^{n}=\frac{\alpha \beta}{R_{e}}, \quad R_{e}=\frac{\rho U_{\infty} L}{\mu_{\infty}}, \alpha=\left(\left(\mu_{0} / \mu_{\infty}\right)-1\right)$,

$\beta=\left(\left(U_{\infty} / L\right) \theta\right)^{n-1}, \quad \tilde{\epsilon}=\hat{\epsilon}^{n}$,

and the leading order velocity fluctuations are considered to be of the order of the friction velocity.

As before, the asymptotic structure of the flow can be found through the single limit concept of Kaplun. This can be easily made through substitution of Eq. (24) into Eq. (36) and passage of the formal limits as the order class of $\eta$ changes.

For the inner flow region the result is,

$\operatorname{ord} \epsilon^{2}=\operatorname{ord} \eta: \overline{\hat{u}} \frac{\partial \overline{\hat{u}}}{\partial x}+\overline{\hat{v}} \frac{\partial \overline{\hat{u}}}{\partial y_{\eta}}=-\frac{\partial \overline{\hat{p}}}{\partial x}-\frac{\partial \overline{\hat{u}^{\prime} \hat{v}^{\prime}}}{\partial y_{\eta}}$,

$\operatorname{ord}(\epsilon \tilde{\epsilon})<\operatorname{ord} \eta<$ ord $\epsilon^{2}: \frac{\partial \overline{\hat{u}^{\prime} \hat{v}^{\prime}}}{\partial y_{\eta}}=0$,

ord $(\epsilon \tilde{\epsilon})=\operatorname{ord} \eta$ :

$$
-\frac{\partial \overline{\hat{u}^{\prime} \hat{v}^{\prime}}}{\partial y_{\eta}}+\frac{\partial}{\partial y_{\eta}}\left(\left(\frac{\partial \overline{\hat{u}}}{\partial y_{\eta}}\right)^{n}+\frac{n(n-1)}{2}\left(\frac{\partial \overline{\hat{u}}}{\partial y_{\eta}}\right)^{n-2} \overline{\left(\frac{\partial \hat{u}^{\prime}}{\partial y_{\eta}}\right)^{2}}\right)=0,
$$

$\operatorname{ord}(\epsilon \tilde{\epsilon} \hat{\epsilon})<\operatorname{ord} \eta<\operatorname{ord}(\epsilon \tilde{\epsilon})$ :

$$
\frac{\partial}{\partial y_{\eta}}\left(\left(\frac{\partial \overline{\hat{u}}}{\partial y_{\eta}}\right)^{n}+\frac{n(n-1)}{2}\left(\frac{\partial \overline{\hat{u}}}{\partial y_{\eta}}\right)^{n-2} \overline{\left(\frac{\partial \hat{u}^{\prime}}{\partial y_{\eta}}\right)^{2}}\right)=0,
$$

$\operatorname{ord}(\epsilon \tilde{\epsilon} \hat{\epsilon})=\operatorname{ord} \eta$ :

$$
\frac{\partial}{\partial y_{\eta}}\left(\left(\frac{\partial \overline{\hat{u}}}{\partial y_{\eta}}\right)^{n}+\frac{n(n-1)}{2}\left(\frac{\partial \overline{\hat{u}}}{\partial y_{\eta}}\right)^{n-2} \overline{\left(\frac{\partial \hat{u}^{\prime}}{\partial y_{\eta}}\right)^{2}}\right)+\left(\frac{\partial^{2} \hat{\hat{u}}}{\partial y^{2}}\right)=0,
$$

ord $\eta<\operatorname{ord}(\epsilon \tilde{\epsilon} \hat{\epsilon}):\left(\frac{\partial^{2} \hat{\hat{u}}}{\partial y^{2}}\right)=0$.

The implication is that for a Carreau fluid, the morphological structure of the turbulent boundary layer is more complex: three principal equations appear in the analysis, Eqs. (38), (40) and (42), characterizing a three-layered structure. 
The contribution of the viscous terms are of leading order in region ord $\epsilon \tilde{\epsilon}>$ ord $\eta$. The power-law viscosity contribution prevails in domain ord $\epsilon \tilde{\epsilon}>$ ord $\eta>$ ord $\epsilon \tilde{\epsilon} \hat{\epsilon}$. In the innermost sub-layer, ord $\epsilon \tilde{\epsilon} \hat{\epsilon}>$ ord $\eta$, the flow behavior is exactly that of a Newtonian fluid.

Thus, for a Carreau fluid, the relevant characteristic lengths in the wall region are:

- Turbulent layer thickness, $\delta=\left(u_{*}^{2} / U_{\infty}^{2}\right) L$.

- Power-law sub-layer thickness, $\tilde{\delta}=\left(K u_{*}^{n-2} / \rho\right)^{1 / n}$, with $K=\left(\mu_{0}-\mu_{\infty}\right) \theta^{n-1}$.

- Newtonian sub-layer thickness, $\hat{\delta}=\left[\left(\left(\mu_{0}-\mu_{\infty}\right) / \mu_{\infty}\right)\right.$ $\left.\theta^{n-1} u_{*}^{n-1}\right]^{1 /(n-1)}$.

The above prescribed order of magnitude between parameters resulted in a rich, three-layered structure. For different limiting cases, different structures can result. For example, in the particular case $\operatorname{ord}(\tilde{\epsilon})=\operatorname{ord}(\hat{\epsilon})$, the power-law term is not capable of playing a dominant role anywhere in the domain and the structure reduces to a classical two-deck Newtonian structure.

\section{Law of the wall: Carreau-Yasuda model}

The above mentioned scarcity of data for purely viscous fluids, made it very difficult to find appropriate experiments to be used in the validation of the boundary layer asymptotic structure for a Carreau fluid.

The work of Japper-Jaafar et al. [18] studies the dynamic behaviour of several water solutions of scleroglucan. Their viscosities are modelled through a Carreau-Yasuda model with power-law indexes ranging from 0.3 to 0.9 . In all, the Carreau-Yasuda parameters for thirteen different solutions are presented. However, mean flow and turbulent statistics are only given for the two lowest concentrations $(0.005$ and $0.01 \mathrm{c} \%(\mathrm{w} / \mathrm{w}))$.

The data of Japper-Jaafar et al. [18] exhibit drag reduction of the order of $15 \%$ and $25 \%$, respectively, for the two lowest concentrations. These effects cannot be solely justified through changes in the shear-thinning behavior and are usually considered to be affected by elasticity. However, in view of the shortage of available data, they are here used to illustrate the contents of the previous section.

For large shear rates, $(\lambda \dot{\gamma})^{a} \gg 1$, the Carreau-Yasuda model (as introduced in [18]) reduces to

$\tau=\mu_{\infty} \frac{\partial u}{\partial y}+\left(\mu_{0}-\mu_{\infty}\right) \lambda^{-n^{\prime}}\left(\frac{\partial u}{\partial y}\right)^{1-n^{\prime}}$.

This equation has a behavior similar to the Carreau model with parameters $\lambda$ and $1-n^{\prime}$ playing the roles of parameters $\theta$ and $n$ (Eq. 32). Essentially, the asymptotic structure of the boundary layer for a Carreau model or a Carreau-Yasuda model is the same.

Eq. (44) suggests three possible definitions for the wall inner variables. In the infinite-shear viscosity limit, the Newtonian term dominates, implying that $y_{\infty}^{+}=y u_{*} / v_{\infty}$. For a combination of shear rates and zero-shear viscosity effects, the non-Newtonian term may give the leading contribution, resulting in $y_{p}^{+}=y u_{*} / v_{p}=y /\left(K u_{*}^{n-2}\right)^{1 / 2}$ with $K=\left(\mu_{0}-\mu_{\infty}\right) \lambda^{-n^{\prime}}$ and $n=1-n^{\prime}$. When both effects are of the same order, $y_{w}^{+}=y u_{*} / v_{w}$, where $\mu_{w}=\mu_{\infty}+\left(\mu_{0}-\mu_{\infty}\right) \lambda^{-n^{\prime}}$ $(\partial u / \partial y)^{-n^{\prime}}$, at $y=0$. Of course, variable $y_{w}^{+}$includes both limiting cases and hence should be used generally.

Fig. 4 shows mean velocity data of [18] plotted in terms of the inner flow variables $y_{w}^{+}=y u_{*} / v_{w}$ and $y_{\infty}^{+}=y u_{*} / v_{\infty}$. The differences are easily spotted but not very pronounced. This is an indication that $v_{w}$ is not far from the infinite-shear viscosity limit.

The viscous region is clearly seen in Fig. 4a; all velocity profiles tend to collapse onto a single curve. The existence of a logarithmic
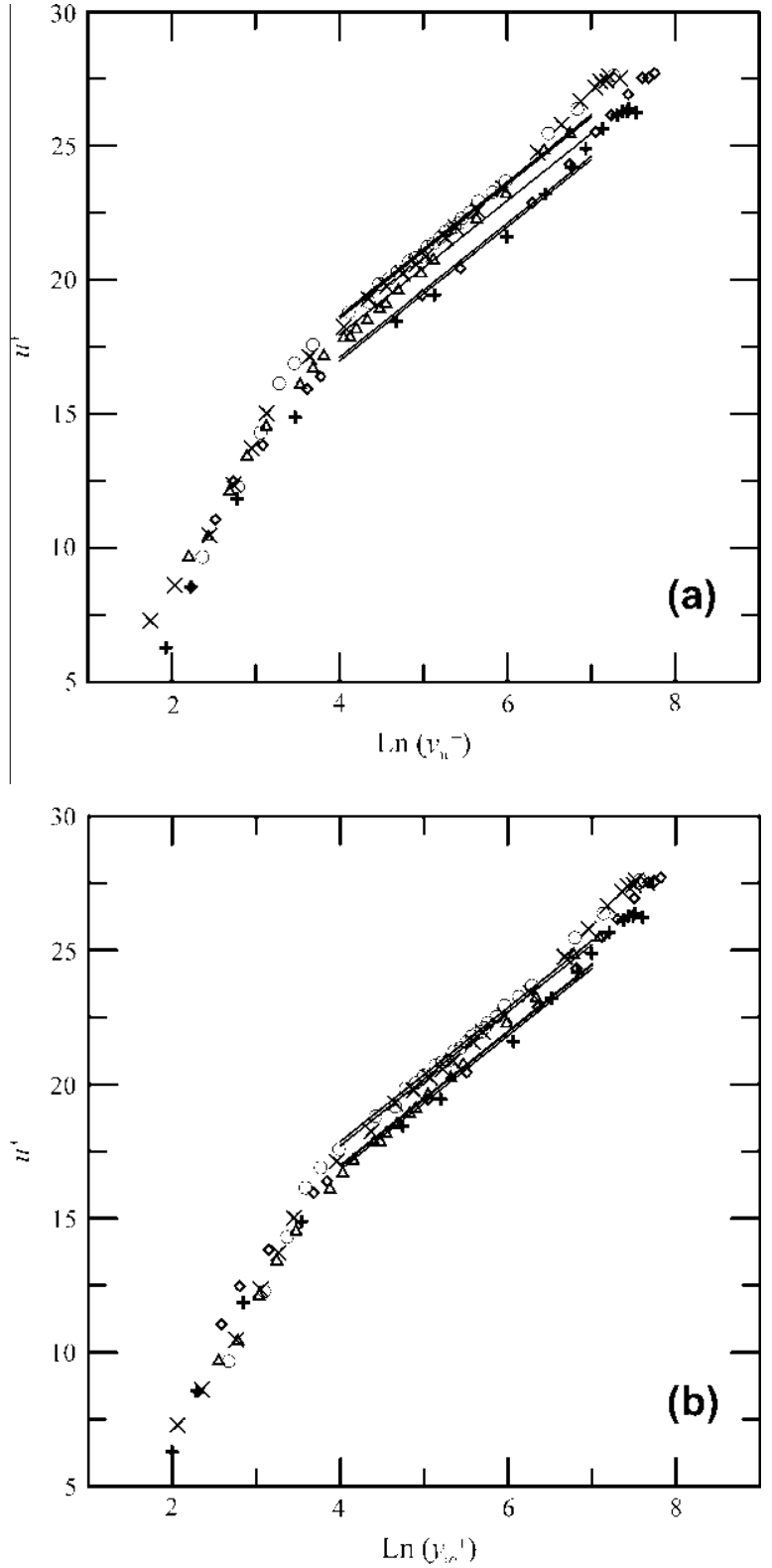

Fig. 4. Mean velocity profiles in wall coordinates according to the data of JapperJaafar et al. [18]. (a) $\left(y_{w}^{+}=y u_{*} / v_{w}\right)$ and (b) $\left(y_{\infty}^{+}=y u_{*} / v_{\infty}\right)$.

region is also visible in Fig. 4. The level of the straight lines are relatively clustered around two values, $7(0.005 \% \mathrm{SG})$ and $8(0.01 \% \mathrm{SG})$. These values are not too removed from predictions furnished by equation $B_{n}=4 / n(=5.71)$. In particular, if a relation based on the DNS data of Rudman et al. [19] is considered, predictions become very good with $B_{n}=7.14$.

For the sake of completeness, the data of Japper-Jaafar et al. [18] is presented in terms of $y_{p}^{+}\left(=y u_{*} / v_{p}\right)$ (Fig. 5). Logarithmic regions are still clearly observable but with very distinct levels. In the viscous region the profile do not collapse onto a single curve, indicating the inadequacy of the choice.

Values of $B_{n}$ for the three different non-dimensional choices are presented in Table 1.

\section{Final remarks}

The asymptotic structure of the turbulent boundary layer of purely viscous non-Newtonian fluids described by the power-law 


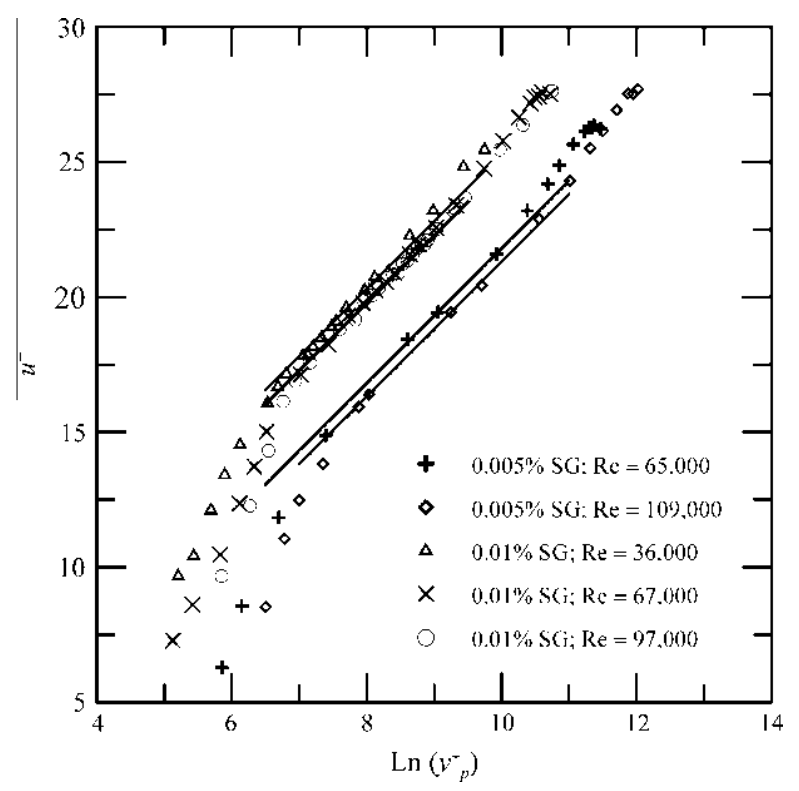

Fig. 5. Mean velocity profiles in power-law coordinates $y_{p}^{+}=y u_{*} / v_{p}=y /\left(K u_{*}^{n-2}\right)^{1 / 2}$ with $K=\left(\mu_{0}-\mu_{\infty}\right) \lambda^{-n^{\prime}}$ and $n=1-n^{\prime}$, according to the data of Japper-Jaafar et al. [18].

Table 1

Behaviour of $B_{n}$ according to the data of Japper-Jaafar et al. [18].

\begin{tabular}{cllll}
\hline Reynolds number & $n$ & $\left(B_{n}\right)_{w}$ & $\left(B_{n}\right)_{\infty}$ & $\left(B_{n}\right)_{p}$ \\
\hline 65,000 & 0.30 & 6.97 & 6.80 & -3.2 \\
109,000 & 0.30 & 7.10 & 6.94 & -3.7 \\
36,000 & 0.31 & 7.95 & 6.98 & 0.30 \\
67,000 & 0.31 & 8.56 & 7.70 & -0.21 \\
97,000 & 0.31 & 8.65 & 7.86 & -0.25 \\
\hline
\end{tabular}

and Carreau viscosity equations have been studied in detail. For power law fluids, the thickness of the viscous sublayer has been shown to depend on the index of power-law models, $n$, through $\hat{\delta}=\left(K u_{*}^{n-2} / \rho\right)^{1 / n}$. The existence of a logarithmic region where the dominant effects are provided solely by turbulence fluctuating terms is also suggested. Changes in $\hat{\delta}$ with $n$ modify the near wall damping effects, altering the level of the logarithmic law, $B_{n}$. Dodge and Metzner [21] suggested Eq. (6) to represent the functional behaviour of $B_{n}$. Despite its complexity, we have shown that predictions for the slope of the log-law do not correspond to evidence obtained from local experimental data. For purely viscous nonNewtonian fluids, the slope of the log-law is constant and equal to 2.5 .

Following suggestions by Clapp [24] and Bogue and Metzner [15], a simple expression for $B_{n}$ can be written in terms of the reciprocal of $n$ according to $B_{n}=4 / n$. Constant 4 is found to be a reasonably good fit to the data of Clapp [24], Bogue and Metzner [15], Escudier and Presti [16] and Pereira and Pinho [17].

However, the DNS data of Rudman et al. [19] suggest

$\frac{u}{u_{*}}=2.5 \ln \left(\frac{y}{\left(K u_{*}^{n-2} / \rho\right)^{1 / n}}\right)+\frac{5}{n}$

This expression has the advantage of naturally reducing to the Newtonian case as $n$ tends to unity.

The dependence of $B_{n}$ on $n$ and the local Reynolds number is a problem that still deserves much consideration. From the data that were available to the present authors, no conclusive judgment could really be made. The natural conclusion is that many more data are still needed so that an irrefutable verdict can be drawn on the true behavior of $B_{n}$.

The integration of Eq. (45) over the cross-sectional area together with some algebraic manipulation yields the following resistance law for the flow in a smooth pipe

$\frac{2 \sqrt{2}}{\sqrt{f}}=2.5 \ln \left(2^{\frac{n-6}{2 n}} f^{\frac{2-n}{2 n}} N_{D}^{\frac{1}{n}}\right)+\frac{5}{n}-3.75$,

where $f$ is the friction coefficient $\left(=8 u_{*}^{2} / U_{\infty}^{2}\right)$.

In fact, the constant 3.75 must be adjusted experimentally; for a Newtonian flow, Nikuradse [30] showed it to be 4.07 .

Results provided by Eq. (46) will be discussed elsewhere.

The correct characterization of the two-layered structure of turbulent boundary layers of non-Newtonian systems has an important impact on near wall turbulence modeling. Indeed, any near wall turbulence model should conform to the correct scales, $u_{*}$ and $\ell$. That is to say that if low-Reynolds number approaches are considered, existing damping functions should be regulated by $u_{*}$ and $\ell$.

\section{Acknowledgments}

In the course of this research, JBRL benefited from a CNPq Research Fellowship (Grant No. 301172/2010-2) and from further financial support through Grant 477354/2011-4. APSF is grateful to the Brazilian National Research Council (CNPq) for the award of a Research Fellowship (Grant No. 303982/2009-8). The work was financially supported by CNPq through Grant No. 477293/ 2011-5 and by the Rio de Janeiro Research Foundation (FAPERJ) through Grant E-26/102.937/2011. Authors have benefited from many useful discussions with Drs. F. P. Duda, A. G. Sousa and L. Fialho. Some of the experimental data have been kindly provided by Prof. F. T. Pinho. In the analysis of the Carreau model, Dr. D. O. A. Cruz made valuable comments that ultimately led to results of Sections 9 and 10 .

\section{References}

[1] A.A. Townsend, The turbulent boundary layer, in: H. Gortler (Ed.), Boundary Layer Research, Springer-Verlag, Berlin, 1958.

[2] H.L. Grant, The large eddies of turbulent motion, J. Fluid Mech. 4 (1958) 149.

[3] S.J. Kline, W.C. Reynolds, F.A. Schraub, P.W. Rundstadler, The structure of turbulent boundary layers, J. Fluid Mech. 30 (1967) 741-772.

[4] L.S.G. Kovasznay, Structure of the turbulent boundary layer, Phys. Fluids Suppl. (1967) S25-S30.

[5] L. Prandtl, Über die ausgebildete turbulenz, ZAMM 5 (1925) 136-139.

[6] Th. von Kármán, Mechanische aehnlichkeit und turbulenz, in: Proc. Third Intern. Congress for Appl. Mech. Stockholm, 1930.

[7] K.S. Yajnik, Asymptotic theory of turbulent shear flow, J. Fluid Mech. 42 (1970) 411-427.

[8] G.L. Mellor, The large Reynolds number, asymptotic theory of turbulent boundary layers, Int. J. Eng. Sci. 10 (1972) 851-873.

[9] W.B. Bush, F.E. Fedell, Asymptotic analysis of turbulent channel flow and boundary-layer flow, J. Fluid Mech. 56 (1972) 657.

[10] V.V. Sychev, V.V. Sychev, On turbulent boundary layer structure, P.M.M.U.S.S.R. 51 (1987) 462-467.

[11] D.O.A. Cruz, A.P. Silva Freire, On single limits and the asymptotic behaviour of separating turbulent boundary layers, Int. J. Heat Mass Transfer 41 (1998) 2097-2111.

[12] J.B.R. Loureiro, A.P. Silva Freire, Scaling of turbulent separating flows, Int. J. Eng. Sci. 49 (2011) 397-410.

[13] S. Kaplun, Fluid Mechanics and Singular Perturbations, Academic Press, 1967.

[14] P.A. Lagerstrom, R.G. Casten, Basic concepts underlying singular perturbation techniques, SIAM Review 14 (1972) 63-120.

[15] D.C. Bogue, A.B. Metzner, Velocity profiles in turbulent pipe flow, Ind. Eng. Chern. (Fund.) 2 (1963) 143-149.

[16] M.P. Escudier, F. Presti, Pipe flow of a thixotropic fluid, J. Non-Newt. Fluid Mech. 62 (1996) 291-306.

[17] A.S. Pereira, F.T. Pinho, Turbulent pipe flow of thixotropic fluids, Int. J. Heat Fluid Flow 23 (2002) 36-51.

[18] A. Japper-Jaafar, M.P. Escudier, R.J. Poole, Turbulent pipe flow of a dragreducing rigid "rod-like" polymer solution, J. Non-Newt. Fluid Mech. 161 (2009) 86-93. 
[19] M. Rudman, H.M. Blackburn, L.J.W. Graham, L. Pullum, Turbulent pipe flow of shear-thinning fluids, J. Non-Newtonian Fluid Mech. 118 (2004) 33-48.

[20] A.B. Metzner, J.C. Reed, Flow on non-Newtonian fluids - correlation of the laminar, transition and turbulent-flow regimes, A.I.Ch.E.J. 1 (1955) 434-440.

[21] D.W. Dodge, A.B. Metzner, Turbulent flow of non-Newtonian systems, A.I.Ch.E.J. 5 (1959) 189-204.

[22] C.B. Millikan, A critical discussion of turbulent flow in channels and tubes, in: Proc. 5th Int. Congress on Applied Mechanics, J. Wiley, N.Y., 1939.

[23] A.H.P. Skelland, Non-Newtonian Flow and Heat Transfer, John Wiley and Sons, New York, 1967

[24] R.M. Clapp, Turbulent heat transfer in pseudoplastiv non-Newtonian fluids, International Developments in Heat Transfer, Part III, A.S.M.E., New York (1961) 652-661.
[25] Y. Tomita, A study of non-Newtonian flow in pipes, Bull. of J.S.M.E. (Jpn Soc Mech. Eng.) 2 (1959) 10-16.

[26] P.A. Lagerstrom, Matched asymptotic expansions, Springer-Verlag, Heidelberg, 1988.

[27] R.E. Meyer, On the approximation of double limits by single limits and the Kaplun extension theorem, J. Inst. Math. Appl. 3 (1967) 245-249.

[28] J.B.R. Loureiro, A.S. Monteiro, F.T. Pinho, A.P. Silva Freire, Water tank studies of separating flow over rough hills, Boundary-Layer Meteorol. 129 (2008) 289308.

[29] J.B.R. Loureiro, A.P. Silva Freire, Note on a parametric relation for separating flow over a rough hill, Boundary-Layer Meteorol. 131 (2009) 309-318.

[30] J. Nikuradse, Gesetzmässigkeiten der turbulenten Strömung in glatten Rohren, Forschg. Arb. Ing.-Wes 356 (1932). 